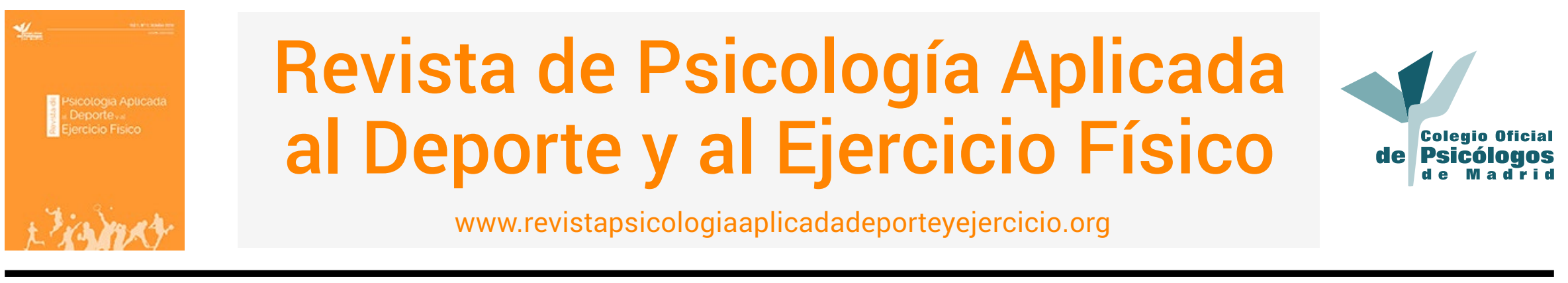

\title{
Variables psicológicas influyentes en el desempeño de árbitros FIFA: un estudio cualitativo
}

\author{
Benito Urra Tobar ${ }^{a, b}$, Claudia Nuñez Corrales ${ }^{b}$, Jorge Oses Lillo ${ }^{b}$ y Gabriela Sarmiento Droguett ${ }^{b}$ \\ aUniversidad Bernardo O'Higgins, Chile \\ bUniversidad Autónoma de Chile, Chile
}

\begin{abstract}
RESUMEN: El presente estudio describe los principales factores psicológicos demandados en el ejercicio del arbitraje desde la percepción de árbitros de la Asociación Nacional de Fútbol Profesional de Chile (ANFP). Para ello, se realizó un estudio cualitativo en donde participaron ocho árbitros internacionales FIFA quienes, a través de entrevistas semi-estructuradas, analizaron la influencia del factor psicológico en su labor. Los resultados indican que la variable mental más mencionada por los participantes fue el "autocontrol" el que puede asociarse al proceso de autorregulación emocional y conductual durante el desempeño. Junto a dicha variable se mencionan otras tales como la concentración, la capacidad de abstracción y el control del partido. Se concluye que los participantes reconocen la influencia del factor psicológico en su desempeño tendiendo a asociarlo a factores emocionales (autocontrol) que influyen en factores cognitivos (concentración) y contextuales dirigidos a un fin central ligado al manejo del juego. Desde los resultados, se discuten las implicancias y posibilidades de intervención en la formación y asesoramiento a árbitros ligadas, principalmente, al desarrollo de estrategias de afrontamiento a las demandas propias del rol de árbitro.
\end{abstract}

PALABRAS CLAVE: fútbol, árbitros, psicología deportiva, variables mentales.

\section{Psychological variables affecting performance of FIFA referees: a qualitative study}

\begin{abstract}
This study describes the main psychological factors required for the practice of refereeing, as perceived by the referees of the "Asociación Nacional de Fútbol Profesional de Chile" (ANFP). For this, a qualitative study was conducted where eight international FIFA referees analyzed, through semi-structured interviews, the impact of the psychological factor on their work. Results show that the most commonly mentioned mental variable was "self-control", which may be associated to the emotional self-regulation and behavioral process during their performance. Other variables reported included concentration, capacity for abstraction, and game control. It is concluded that participants recognize the influence of psychological factors in their performance and tend to associate it to emotional factors (self-control) that affect cognitive (concentration) and contextual factors directed to a central objective related to game management. Based on the results, the implications and the possibilities for interventions in referee training and advising, mainly intended to develop strategies to face the demands inherent to the role of the referee, are discussed.
\end{abstract}

KEYWORDS: Football, Referee, Sport Psychology, Mental Variables.

\section{Variáveis psicológicas que influenciam o desempenho dos árbitros da FIFA: um estudo qualitativo}

RESUMO: O presente estudo descreve os principais fatores psicológicos exigidos no exercício da arbitragem a partir da perceção de árbitros da Associação Nacional de Futebol Profissional do Chile (ANFP). Para tal, foi realizado um estudo qualitativo no qual participaram oito árbitros internacionais da FIFA, os quais através de entrevistas semiestruturadas, analisaram a influência do fator psicológico no seu trabalho. Os resultados indicam que a variável mental mais mencionada pelos participantes era "o autocontrolo", o

\footnotetext{
Benito Urra Tobar es Psicólogo y docente en la Universidad Bernado O'Higgins y la Universidad Autónoma de Chile; Claudia Núñez Corrales es Psicóloga y docente en la Universidad Autónoma de Chile; Jorge Oses Lillo es Psicólogo y docente en la Universidad Autónoma de Chile; Gabriela Sarmiento Froguett es Psicóloga y docente en la Universidad Autónoma de Chile.
} 
qual pode ser associado ao processo do autorregulamento emocional e comportamental durante o desempenho. Outras variáveis são mencionadas como a concentração, a capacidade de abstração e o controlo do jogo. Conclui-se que os participantes reconhecem a influência do fator psicológico no seu desempenho que tende a associá-lo aos fatores emocionais (autocontrolo) que influenciam os fatores cognitivos (concentração) e contextuais dirigidos a um alvo central associado à manipulação do jogo. A partir dos resultados, discutem-se as implicações e possibilidades de intervenção na formação e aconselhamento de árbitros associadas, principalmente, ao desenvolvimento das estratégias para enfrentar as próprias exigências do papel de árbitro.

PALAVRAS-CHAVE: futebol, árbitros, psicologia desportiva, variáveis mentais.

Artículo recibido:-24/08/2018 | Artículo aceptado: 15/10/2018

El fútbol es uno de los deportes con mayor popularidad e impacto social, económico y cultural a nivel mundial (García-Naveira, 2018). Dado el creciente desarrollo del fútbol, se hizo necesaria la implantación de normas reglamentarias y, con ello, surge la figura del árbitro como agente representativo del carácter regulado y competitivo del juego, debiendo velar por el correcto cumplimiento de las reglas del mismo (Pedrosa y García-Cueto, 2015).

La complejidad del rol de árbitro se expresa en la variedad de funciones que deben cumplir: percibir, evaluar, decidir y sancionar para hacer cumplir las leyes del juego, prestar atención al juego para tomar decisiones y realizar juicios durante el partido en tiempos muy inmediatos y breves (Di Corrado, Pellarin y Agostini, 2011; González, 2006; González, Selva y Torregrossa, 2018).

La labor del árbitro está sometida a exigencias ligadas al desempeño, presiones internas (metas, temores) y externas (medios, público, entrenadores, dirigentes, etc.) y factores socioeconómicos. Al respecto, Slack, Maynard, Butt y Olusoga (2013), a través de un análisis inductivo de contenidos realizados a la información proporcionada por 15 árbitros, reportan la existencia de necesidades físicas, ambientales (ligadas al manejo efectivo del juego: respeto, comunicación) y psicológicas (manejo de estrés, resiliencia, características personales).

La complejidad del arbitraje deportivo se ve acentuada por la constante evaluación del desempeño por parte de agentes que cuentan con recursos tecnológicos y enfatizan el error más que en el acierto tornándolo una figura polémica frecuentemente expuesta a críticas y/o reacciones emocionales (Mascarenhas, Collins y Mortimmer, 2005).

A pesar del protagonismo que tienen y la alta tensión que soportan durante su actuación, los árbitros de fútbol han sido poco visibilizados por la literatura científica en psicología deportiva, recomendándose una mayor atención tanto en el ámbito aplicado como el de investigación (García-Naveira, 2018; González et al., 2018).
De tal forma, es posible apreciar estudios que analizan el impacto en el desempeño arbitral de factores como la personalidad (Pla-Cortés, Gomá-i-Freixanet y Avilés-Antón, 2015), motivación (Alonso-Arbiol, Arratibel y Gómez, 2008), estrés (Froeseler, Monteiro y Mansur-Alves, 2018; García-Santos, Vaquera, Calleja-González, González-Espinoza e Ibañez, 2017; Vela y Arbinaga, 2018), burnout (Al-Haliq, Lufti y Oudat, 2014) y ansiedad (Tore y Haugen, 2013).

Ello revela que, si bien el arbitraje deportivo involucra una serie de conocimientos, agudeza visual y condiciones físicas, también se ve influido por recursos psicológicos y comunicativos que requieren ser potenciados a través de la preparación psicológica (Mathers y Brodie, 2015; Pedrosa y García-Cueto, 2015). Al respecto, Guillén y Jiménez (2001), empleando una muestra de 247 árbitros de 21 deportes, identifican como características deseables del arbitraje deportivo la imparcialidad/justicia, el conocimiento y aplicación del reglamento, la honradez y la igualdad de trato a los deportistas. En términos de habilidades mentales específicas se reportan la capacidad de atención y concentración, la personalidad/carácter, la autoridad/disciplina y el ser comunicativo/dialogante.

Asimismo, Weinberg y Richardson (1990) señalan que los mismos árbitros identifican destrezas psicológicas (concentración, confianza, decisión, comunicación interpersonal y control emocional) que diferencian a los árbitros más competentes y que son responsables del $50 \%$ al $70 \%$ del éxito arbitral.

En el ámbito del fútbol, Villalobos, Ugarte y Guillén (2002) reportan, en un estudio realizado con 58 árbitros, que la mayoría de las cualidades mencionadas como deseables en el árbitro se refieren a factores de carácter psicológico (73\%) y que los defectos auto-atribuidos se refieren a factores de personalidad (87.70\%) por sobre factores físicos y técnicos (12.30\%) siendo éstos últimos los más trabajados en la formación. 
Para Blumenstein y Orbach (2014) las principales variables mentales asociadas al arbitraje son el manejo del estrés y la presión, la autoconfianza, la resolución de conflictos y la concentración frente a distractores como los errores, la baja autoconfianza, la alta ansiedad por la evaluación y el mal ánimo antes del juego. Asimismo, Peirooz (2013) considera que las principales variables mentales que inciden en el desempeño del árbitro son el manejo del estrés, la toma de decisiones, el manejo de los factores sociales y la concentración.

Finalmente, Schnyder y Hossner (2016) reportan, en una muestra de 23 árbitros de fútbol, que un buen árbitro debe tener conocimiento del deporte, habilidades de manejo del juego como el liderazgo y el manejo del contexto $(n=21)$, atributos mentales $(n=18)$, características personales asociadas al rol $(n=17)$, inteligencia futbolística $(n=15)$ y experiencia $(n=6)$. En términos específicos de las habilidades mentales se menciona una condición mental óptima ( $n=$ 11), concentración $(n=6)$, autoconfianza $(n=6)$ y control emocional $(n=1)$.

La evidencia empírica expuesta sugiere que el estrés aparece como uno de los factores más relevantes en el desempeño arbitral pues la toma de decisiones de los árbitros está influida por las emociones dentro de contextos de tensión que ponen en riesgo el control y capacidad de juicio perceptivo que el árbitro debe aplicar durante el juego (Márquez, 2005). Además, el árbitro se enfrenta a otras fuentes de estrés tales como los errores, la ocurrencia de lesiones, el miedo al fracaso, evaluaciones deficientes, no cumplimiento de expectativas, la falta de reconocimiento y respeto, conflictos interpersonales, la presión del tiempo (Al-Haliq et al., 2014), la responsabilidad propia del rol (Samuel, Galily y Tenenbaum, 2015) y el conflicto entre familia y arbitraje (Voight, 2009), existiendo evidencia que sugiere que los estresores sociales son más relevantes que los estresores físicos (Froeseler et al., 2018).

Por ello, es necesario que éste desarrolle la capacidad de autocontrol para el manejo del estrés frente a errores, conflictos interpersonales y agresiones verbales de jugadores, entrenadores y público (Anshel, Kang y Jubenville, 2013). Entonces, resulta relevante el entrenamiento en estrategias de afrontamiento para la correcta gestión de tensiones emocionales excesivas durante la competencia (Samuel, Galily y Tenenbaum, 2015; Wolfson y Neave, 2007).

Respecto a la atención y concentración, Weinberg y Richardson (1990) plantean que esta capacidad resulta fundamental en un árbitro ya que debe diferenciar los estímulos relevantes de aquellos secundarios, en escenarios de alta complejidad y marcados por situaciones sorpresivas y de incertidumbre.
De tal forma, es posible mencionar una asociación entre "lo psicológico" con el ámbito emocional (ansiedad, manejo de estrés, autocontrol, ánimo), sumándose habilidades de carácter cognitivo (autoconfianza, concentración) e interpersonales (manejo del juego, comunicación, control ambiental). Por consiguiente, en el arbitraje la manera de experimentar los procesos mentales implicados en el desempeño puede producir activaciones emocionales que inciden en la conducta y las expectativas de éxito (Brandão, Serpa, Krebs, Araújo, y Machado, 2011). En tal sentido, se ha planteado la importancia de las creencias de eficacia de los árbitros, esto es, la medida en que creen que tienen la capacidad de desempeñar con éxito su trabajo, generando seguridad en sus actuaciones y decisiones a pesar de un mal desempeño (Guillén y Feltz, 2011).

La intervención psicológica destinada a la optimización del desempeño en el arbitraje deportivo es reconocida actualmente como un campo relevante de aplicación de la disciplina por cuanto se ha planteado que es posible considerar al árbitro como un atleta de alto rendimiento (Froeseler, Monteiro y Mansur-Alves, 2018); sin embargo, pese a dicha valoración profesional y académica, son pocos los árbitros que recurren al asesoramiento psicológico (Ramírez, Alonso-Arbiol, Falcó y López, 2006).

Ello se refleja en que los aspectos psicológicos tienden a estar ausentes en la formación de los árbitros, pese a que los factores mentales aparecen como una de las principales deficiencias en el desempeño (Mascarenhas et al., 2005). En tal sentido, una adecuada preparación mental de los árbitros de fútbol permitiría trabajar aspectos psicológicos relacionados con su labor que facilitarían su desempeño.

Sin embargo, un esquema de intervención requiere de una instancia previa de un acercamiento empírico para detectar las necesidades psicológicas de los árbitros de fútbol en base a su experiencia para después aplicarlos a situaciones prácticas en base al conocimiento obtenido (Peris-Delcampo y Cantón, 2018; Schnyder y Hossner, 2016). Para ello, se privilegia el enfoque cualitativo y, más específicamente, el empleo de entrevistas por sobre otros procedimientos de evaluación (observaciones, tests, etc.) por cuanto ella permite profundizar en la experiencia de los sujetos participantes no sólo en términos de los factores identificados sino también en el significado y vivencias asociados a ellos (Guillén, Peñarrubia, Montero y Adell, 2014), más aún considerando que sólo un 3,5\% de los artículos publicados en revistas científicas españolas del ámbito de las ciencias de la actividad física y el deporte utilizan un abordaje cualitativo (Molina, Villamón y úbeda, 2015).

A partir de ello, el objetivo del presente estudio es describir los principales factores psicológicos demandados en 
el ejercicio del arbitraje y la forma en que ellos son experienciados desde la percepción de los árbitros FIFA de la Asociación Nacional de Fútbol Profesional de Chile. Como hipótesis de trabajo se plantea que los árbitros, de acuerdo a la evidencia existente, identificarán como variables psicológicas relevantes en el desempeño arbitral factores emocionales (estrés, ansiedad, autocontrol), cognitivos (autoconfianza, concentración) e interpersonales (manejo del juego, comunicación).

\section{Método}

Se realizó un estudio cualitativo y descriptivo para comprender, a través de las propias experiencias, relatos y significados de los árbitros de fútbol, la manera en que perciben la influencia de los factores mentales en el arbitraje. La metodología cualitativa se ha empleado en Psicología del Deporte (Brewer, Vose, Van Raalte y Petitpas, 2011) y, en dicho contexto, en estudios con árbitros (Alonso-Arbiol, Arratibel y Gómez, 2008; Patiño y Cañadas, 2015). Se utilizó un diseño no experimental, transaccional y fenomenológico, por cuanto, de acuerdo a Guillén et al. (2014) y Rosado et al. (2014), este enfoque representa la mejor vía para analizar las significaciones subjetivas de las personas frente a las situaciones tal cual se presentan, así como la interiorización y representación que hace de ellas para, de esta forma, explorar una realidad desde quienes forman parte de ella.

\section{Participantes}

Se trabajó con una muestra compuesta por 8 árbitros internacionales FIFA varones, con edades entre los 34 y 41 años de edad $(M=38.25 ; D T=2.63)$ y experiencia arbitral profesional entre 5 y 20 años $(M=11.37$ años; $D T=5.87$ ) quienes participaron de manera voluntaria en el estudio. Esta muestra representa el $75 \%$ de los árbitros FIFA varones chilenos y el $65.53 \%$ del total de árbitros FIFA del país.

El muestreo es de tipo homogéneo, esto es, dirigido a una población específica con características similares a fin de estudiarlo a fondo permitiendo generar un análisis simplificado, lograr focalización, obtener variaciones reducidas y facilidades para entrevistas (Heinemann, 2008). Los criterios de inclusión para formar parte de la muestra fueron: 1) ser árbitro de fútbol; 2) pertenecer a la categoría FIFA y 3) aceptar formar parte de la investigación y firmar el consentimiento informado.

Se utilizó como criterio de inclusión la pertenencia al grupo de Árbitros Internacionales FIFA por cuanto ello implica la participación en partidos de alta exigencia que requieren habilidades mentales aplicadas al deporte, así como cumplir una serie de requerimientos implicados en una calificación que define cambios en la carrera del sujeto (Samuel, Galily y Tenenbaum, 2015).

Finalmente, cabe consignar que también formaron parte del estudio tres expertos en el área temática: un entrenador de fútbol, un ex árbitro profesional y un psicólogo del deporte, con edades entre los 35 y 47 años de edad y amplia experiencia en el ámbito del fútbol (entre los 5 y los 18 años). Los tres sujetos participaron en la fase de validación formal y de contenido de la entrevista. La entrevista fue realizada por el psicólogo del deporte.

\section{Instrumento}

Se empleó una entrevista semi-estructurada, siguiendo los lineamientos de Bohórquez y Checa (2017), realizada cara a cara, no dirigida, de estudio, individual y creada adhoc para este trabajo. Esta entrevista validada previamente por tres expertos con el fin de analizar la validez formal (vocabulario, redacción) y de contenido (organización, pertinencia, temáticas abordadas, aspectos faltantes) de la entrevista empleada.

Protocolo de entrevista. los participantes respondieron a una entrevista individual, y de carácter confidencial, de una duración aproximada de 45 minutos, junto con establecer el rapport necesario para el desarrollo de la entrevista, se les invitó a hablar sobre su percepción acerca del factor psicológico en el arbitraje de fútbol a partir de preguntas guía y no cerradas para explorar eventuales conocimientos emergentes (Schnyder y Hossner, 2016).

Pauta de entrevista semi-estructuradas. se recopiló la información a través de entrevistas que, siguiendo el esquema planteado por Rosado et al. (2015), incluye preguntas destinadas a desarrollar rapport, analizar espectos emergentes y profundizar en los temas analizados para, de esta forma, introducirse en el mundo psicológico del informante. Este enfoque de entrevista es coherente con la metodología fenomenológica en donde el sujeto es considerado como el experto y es el significado asociado a sus experiencias el objeto relevante para el investigador.

Tal como sugieren Bohórquez y Checa (2017), las entrevistas comenzaron con preguntas generales primero referidas a aspectos demográficos y de historia personal, para luego inquirir sobre las categorías indicadas en la Tabla 1. Además, se incluyeron preguntas relativas a la formación, estrategias empleadas y rol del psicólogo del deporte que son parte de un estudio en preparación. 
Tabla 1: Categorías de la entrevista realizada a árbitros FIFA $(n=8)$

\begin{tabular}{ll}
\hline Sesiones & Ejemplo de preguntas \\
\hline Percepción general de la influencia del factor mental en el arbitraje & $\begin{array}{l}\text { ¿Qué importancia le da al factor psicológico en el arbitraje?; (-si siente } \\
\text { que influye-) "iDe qué manera influye lo psicológico en la labor del } \\
\text { árbitro }\end{array}$ \\
\hline $\begin{array}{l}\text { Identificación de los factores psicológicos considerados relevantes } \\
\text { para el arbitraje }\end{array}$ & $\begin{array}{l}\text { ¿Qué factores psicológicos son los más relevantes para el arbitraje?; } \\
\text { ¿Por qué los considera relevantes?) }\end{array}$ \\
\hline $\begin{array}{l}\text { Significados asociados a las variables mentales identificadas } \\
\text { Impacto concreto de los factores mentales identificados sobre el } \\
\text { desempeño del árbitro }\end{array}$ & $\begin{array}{l}\text { ¿Qué significa para usted la variable __? } \\
\text { ¿De qué forma concreta influye el factor __ sobre el desempeño } \\
\text { arbitral?; ¿Qué efectos tiene este impacto en tu desempeño?; ¿Cómo } \\
\text { te das cuenta de la influencia de este factor sobre tu trabajo?) }\end{array}$
\end{tabular}

\section{Procedimiento}

Siguiendo lo expuesto por Bohórquez y Checa (2017), el procedimiento de trabajo se inició con la revisión de la literatura sobre las necesidades psicológicas del arbitraje deportivo y en el fútbol para luego diseñar, de manera deductiva, las categorías de análisis necesarias en el análisis cualitativo. Posteriormente, se diseñó una entrevista piloto, que fue sometida a juicio de expertos (ex árbitro, entrenador y psicólogo del deporte) quienes asignaron una puntuación, de 1 como nada pertinente a 4 como totalmente pertinente, a cada pregunta, atendiendo a la necesidad y atingencia de cada una de ellas. Los resultados indican un acuerdo en las puntuaciones asignadas por los jueces en un del $92 \%$ sobre el total de observaciones realizadas a las preguntas sugeridas (ofrecieron las misma puntuación a los ítems sugeridos).

Tras los resultados del análisis se elaboró la versión final que se adjunta al final del presente trabajo. Después de obtener la autorización del Presidente de la Comisión de Árbitros, se procedió a la realización de las entrevistas previa firma del consentimiento informado, que resguarda las condiciones de anonimato y confidencialidad de las respuestas. Éstas se realizaron en lugares convenidos con los informantes que resultaron finalmente en las oficinas de la ANFP de Chile, facilitando la privacidad, evitación de interferencias y la grabación en audio; asimismo las condiciones ambientales del lugar de entrevista, en términos de temperatura, luz y ruido eran óptimas, por lo cual no se apreciaron limitaciones en esta etapa sino, al contrario, alta participación e interés por el estudio.

\section{Análisis de datos}

Las entrevistas fueron trasncritas literalmente, tras lo cual se realizó un análisis inductivo de contenido con el fin de lograr una descripción objetiva y sistemática de la información en base a la clasificación y/o codificación de los diversos elementos de un mensaje en categorías, para otorgar un significado a los relatos de acuerdo a los objetivos de investigación (Brewer et al., 2011; Guillén et al., 2014). Para ello, las respuestas dadas a las preguntas conformantes de las distintas categorías de análisis fueron codificadas siendo ingresadas literalmente al contenido de las mismas.

\section{Resultados}

A través de las entrevistas realizadas a árbitros FIFA, es posible identificar los factores psicológicos más relevantes que, desde la percepción de los árbitros participantes, influyen, directa o indirectamente, en su desempeño arbitral.

El primer hallazgo que se evidencia es que la variable psicológica con mayor frecuencia de asociación con el desempeño arbitral, y que es mencionada por todos los sujetos entrevistados $(n=8)$, es el autocontrol. Éste es entendido como la capacidad de mantener el dominio de si a pesar del grado de tensión al que se vean expuestos en la práctica, ya que les permite la obtención de un juego equilibrado y de un buen manejo en situaciones conflictivas. En tal sentido, frente a la pregunta referida a en qué momentos concretos influye esta variable, un árbitro comenta: "Lo están presionando, le están gritando, le están diciendo esto, le están diciendo esto otro, si no tiene la capacidad de soportar de tolerar esa presión y los insultos, y todo eso con lo que nosotros convivimos lamentablemente no sirve para esto" (Sujeto 6).

En tal sentido, los árbitros, manifiestan la importancia que posee una adecuada regulación y autocontrol en su actuar durante el partido, principalmente en situaciones ligadas al manejo de presión y estrés implicados en su labor; en tal sentido, cuando se le pide definir y darle una utilidad a esta 
habilidad un árbitro reporta que: "Es tener control de uno mismo, suele pasar que siempre uno está bajo una presión y tiene que actuar siempre de modo racional y no irracional" (Sujeto 1).

Por ello, el equilibrio y adecuado autocontrol permite al árbitro mantener la tranquilidad frente a las críticas de diversos agentes (jugadores, entrenadores, hinchas) asumiendo que sin la capacidad de sobrellevarlas es difícil mantenerse dentro del partido. Esta situación es reportada por 6 de los árbitros participantes, lo cual se refrenda en la siguiente afirmación expresada por uno de los sujetos: "Lo que uno tiene que siempre mantener, es la calma ante todas estas situaciones complicadas" (Sujeto 3).

Una estrategia de autocontrol se halla ligada al foco atencional y se expresa como la capacidad para distraerse de eventos distractores y centrarse en los estímulos relevantes del desempeño. Bajo ese contexto, 5 árbitros mencionan como un factor psicológico relevante en el arbitraje a la "capacidad de abstracción" situándolo como una habilidad en sí misma y no como un medio de orientación de la atención.

La definición de esta variable, dada por 4 árbitros entrevistados, la asocia al modo en que un árbitro de fútbol separa, a través de diferentes actividades, aquello que podría influir negativamente en su desempeño, para que, una vez iniciado el partido, atienda aquellos estímulos atingentes al desarroIlo del juego. En ese sentido, al responder cuándo esta variable resulta influyente un árbitro comenta que: "Antes de un partido importante puede ser, yo lo que intento es estar con la familia no ver fútbol no veo fútbol veo otra cosa, programas de entretenimiento, cosas, pero pensar en partidos no" (Sujeto 4).

Sin embargo, más que como una variable en sí misma, la "capacidad de abstracción" aparece entendida como una estrategia de afrontamiento ligada al manejo de pensamientos y la orientación del foco atencional frente a estímulos interferentes en el desempeño. Ello se refleja en que, frente a la misma pregunta, 3 árbitros asocian la capacidad de abstraerse durante el partido a no centrarse en el error, lo que les permite seguir avanzando y no cometer nuevos errores: "Tienes que abstraerte muchas veces cuando has cometido un tipo de error, cuando sabes, nosotros dentro del campo de juego inmediatamente te das cuenta" (Sujeto 2).

Ligado a ello, otro factor psicológico descrito en el relato de los árbitros es la concentración durante el partido, entendida como el mantenimiento sostenido de la atención durante un partido, logrando actuar de manera óptima ante una situación pues permite ampliar el foco atencional tal como sostiene un entrevistado frente a la pregunta de en qué momento es necesaria esta habilidad: "Cuando viene un pase largo tenemos que buscar la estrategia y la concentración de ver más de lo que podemos ver, ampliar nuestro campo visual" (Sujeto 4).

Los árbitros sostienen que la capacidad de concentración es difícil de mantener, ya que la labor que realizan está expuesta a diferentes situaciones que conlleva a la perdida de la concentración en el transcurso de la actividad: "El mantener una concentración durante todo el partido, que no es algo fácil, ya que hablábamos anteriormente que ciertos estímulos que se producen durante el juego, que nos llevan a desconcentrarnos" (Sujeto 8).

Con respecto a la concentración durante el partido, los árbitros indican que existen situaciones en que la perdida de concentración les afecta en la labor arbitral, por lo que requieren de una estrategia para retomarla. En tal sentido, el mismo árbitro que vincula la concentración a la ampliación del campo visual, vuelve a darle un matiz práctico al impacto de esta variable sobre el desempeño deportivo: "Porque si perdemos la concentración miramos hacia el balón cuando dan el pase y nos enfocamos en el último ese movimiento de cabeza vamos a ver completamente distinto todo lo que pasó" (Sujeto 4)

Finalmente, otro factor psicológico influyente en el desempeño percibido por los árbitros entrevistados $(n=4)$ es lo que denominan como "control del partido", el cual se asocia a la responsabilidad de mantener la normalidad en el comportamiento de los jugadores durante el juego, facilitando el manejo del encuentro y permitiendo una actuación segura, dentro de los márgenes establecidos y delimitando de manera clara las reglas del juego. En tal sentido, al momento de referirse a por qué es relevente esta variable, un árbitro indica: "Porque los jugadores siempre quieren cobrar, cobrar su parte entonces uno tiene que tener la capacidad de poder 'hey, tranquilos muchachos, tranquilos', de volverlo a cierto margen en donde no los puede manejar los puede conducir, y donde puede terminar el partido de buena forma" (Sujeto 3).

Sin embargo, a forma de entender y aplicar este factor, lo hace ver más como un resultado de un adecuado funcionamiento mental más que una variable psicológica en sí misma evidenciando cierta confusión en el modo en que los árbitros conceptualizan la experiencia mental. Ello se refleja en que, a juicio de los árbitros que identificaron esta variable, el control del partido facilita que puedan efectuar su trabajo y resguarden el desarrollo del partido, ya que mantienen la tranquilidad y el control del juego, lo que disminuye los conflictos frente a situaciones determinantes para el resultado del partido: "Como lo llevamos a la calma, como logramos controlar a ese jugador para que no siga eeh eeh sobreactuando dentro del terreno de juego" (Sujeto 5).

Asimismo, el control del partido permite, además, que los árbitros efectúen sus labores con seguridad, ya que sin ésta 
pueden verse sobrepasados por los mismos jugadores; así, frente a la pregunta sobre la importancia de esta "variable mental", un entrevistado comenta que: "Pasa por un tema de inseguridad y eso el jugador lo percibe, está viendo que uno está inseguro y por ahí uno empieza a perder el control" (Sujeto 1). Ello evidencia que el control del partido no es más que un resultado o consecuencia caracterizada por un manejo del contexto consistente en la trasmisión de una imagen a los jugadores que facilite mantener su conducta dentro de los límites reglamentarios.

En resumen, se logra apreciar que las significaciones en torno a los factores psicológicos se encuentran ligados a la concentración y el autocontrol en el arbitraje; en ese contexto, una "variable mental" identificada como capacidad de abstracción aparece como una estrategia que articula ambos factores en tanto obedece a una capacidad atencional que actúa como estrategia de afrontamiento que ayuda, entre otras cosas, a mantener el control emocional. A partir de ello, se espera una consecuencia, que los sujetos catalogan como una variable mental ("control de juego"), actúe como una herramienta de manejo y gestión del entorno. Sin embargo, la forma de entenderla y analizar su impacto revela que, más que un factor mental interno al sujeto, responde a una consecuencia de su accionar que debiese evidenciar un adecuado funcionamiento de otras variables realmente intrinsecas del dinamismo psíquico.

\section{Discusión}

Los resultados obtenidos permiten el logro del objetivo general del estudio por cuanto ellos indican que los árbitros plantean que su labor se ve influida, además de capacidades reglamentarias, técnicas y físicas, por una serie de factores mentales que pueden potenciar o perjudicar su desmepeño. En este contexto, todos los árbitros participantes identifican al autocontrol emocional en el arbitraje como un aspecto psicológico influyente en su desempeño, lo cual obedece a que, según Márquez (2005), esta actividad demanda el constante dominio de sí, regular las emociones y afrontar de manera asertiva las tensiones y situaciones conflictivas que suelen darse en el desarrollo del juego.

Ello permitiría actuar de modo racional y tomar decisiones objetivas y ajustadas al reglamento obteniendo, de esta manera, equilibrio, credibilidad y seguridad en las sanciones permitiendo un manejo efectivo del encuentro. En tal sentido, es posible plantear que el autocontrol aparece, tal como sostienen Wolfson y Neave (2007), como una estrategia de afrontamiento frente al estrés y las demandas propias del rol de árbitro (Samuel, 2015).
Con menor frecuencia de indentificación, pero no por ello menos relevantes, los árbitros también mencionan a la concentración, la capacidad de abstracción y el control del partido. De acuerdo a los resultados obtenidos, sin embargo, estas tres variables aparecen como subordinadas al funcionamiento emocional del árbitro. Esto es, la concentración, la capacidad de abstracción y el control del partido dependen del nivel de autoconciencia y gestión que el árbitro tenga de sus emociones y del modo en que ellas influyen en la competencia: si el árbitro logra mantener un control sobre su estado emocional podrá concentrarse, abstraerse de lo alejado al momento y controlar el juego.

En términos concretos, la importancia de mantener la concentración durante los 90 minutos del juego radica en que, en concordancia con lo planteado por Blumenstein y Orbach (2014) y Schnyder y Hossner (2016), ello permite discriminar entre los aspectos relevantes y aquellos que son perjudiciales en el trabajo arbitral, permitiendo tener mayor claridad y seguridad para realizar juicios y tomar decisiones durante el partido; así, el que un árbitro esté concentrado no garantiza el éxito pero sí aumenta considerablemente la posibilidad de responder adecuadamente ante situaciones inesperadas.

Por su parte, la capacidad de abstracción permite que los árbitros generen un estado mental de equilibrio para afrontar el encuentro deportivo y, por tanto, su rendimiento alcance niveles eficientes. Por ello, resulta relevante que un árbitro cuente con la capacidad de entregar importancia a situaciones del partido que realmente lo requieran, gestionando de forma adecuada elementos que son inherentes a un partido de fútbol, como por ejemplo la alta presión que genera el encuentro, y que puedan influir negativamente en la toma de decisiones.

Si bien la capacidad de abstracción es mencionada, por algunos de los participantes, como una variable psicológica específica puede entendersele como una habilidad íntimamente ligada, sino parte, de la atención por cuanto se asocia a la manera en que el árbitro se separa (es decir, aleja el foco atencional) de aquello que podría influir negativamente en su desempeño, para que durante el partido atienda a los estímulos atingentes al desarrollo del juego.

En este sentido, los aspectos de los que resulta necesario abstraerse pueden obedecer a estresores deportivos, ampliamente expuestos por Al-Haliq et al. (2014) y Anshel, Kang y Jubenville (2013), como extradeportivos en donde resalta, en concordancia lo expuesto por Voight (2009) las consecuencias que el rol arbitral tiene para la vida familiar y que lleva a algunos de los árbitros participantes la necesidad que el psicólogo deportivo indague en su vida personal y los pueda orientar al respecto.

En tercer lugar, los árbitros participantes identifican el con- 
trol/manejo del juego como una variable psicológica siendo que dicho concepto sugiere que, más que ser un factor mental en sí mismo, es posible considerarlo como el resultado de un desempeño influido por aspectos psicológicos. Sin embargo, la descripción de esta variable parece estar asociada a la dimensión de autocnocimiento y evaluación del propio desempeño lo cual podría conceptualizarse como un proceso de índole mental.

En términos concretos, el reporte de los árbitros participantes indica que el control del partido permite hacer una evaluación objetiva de la calidad de su desempeño, manejo de temores y conflictos así como observar aciertos y conductas que demuestran credibilidad, lo cual, de acuerdo a González (2006) desarrollar la autoconfianza en sí mismo y la capacidad de manejar la presión durante la competencia vinculándose, por ello, a las habilidades de comunicación del sujeto frente a los errores y situaciones de alta complejidad en donde se tiene la responsabilidad de mantener la conducta de los jugadores dentro de los márgenes establecidos por las reglas del juego.

Resulta necesario destacar que las variables psicológicas mayormente reportadas por los participantes (autocontrol, concentración y capacidad de abstracción) son vistas no como un fin en sí mismas, sino como un medio para un fin mayor: permitir un buen manejo del juego y del contexto en que éste se lleva (lo que ellos denominan como control del juego). Ello puede explicarse porque, según Di Corrado et al. (2011) y Vela y Arbinaga (2018), el desempeño arbitral depende de la forma de responder a las claves contextuales, lo cual hace aparecer como habilidades deseables el autocontrol/regulación emocional y el liderazgo/autoridad en tanto herramientas de gestión del ambiente y manejo del juego (Márquez, 2005; Unkelbach y Memmert 2008)

Sin embargo, la evidencia previa expone la existencia de otros aspectos psicológicos a considerar para lograr un óptimo desempeño en un árbitro, tales como factores perceptivos (Abbedaim, Sebbane, Zitouni, Zubiaur y Boumesdji, 2016; Schnyder y Hossner, 2016), autoconfianza, habilidades comunicativas y toma de decisiones (Guillén y Feltz, 2011; Peirooz, 2013). No obstante, estos aspectos psicológicos no fueron mencionados por los participantes de este estudio, razón que permite evidenciar que los árbitros de la ANFP no consideran como prioridad dichos factores psicológicos, por cuanto el autocontrol engloba la autoconfianza y la efectividad de la comunicación con el equipo arbitral.

Entonces, si bien los resultados obtenidos permiten comprobar la hipótesis del estudio, por cuanto los participantes describen factores psicológicos de carácter emocional ("autocontrol"), cognitivo ("concentración") y sociales/interpersonales ("control del juego"), es posible apreciar que lo psi- cológico parece estar ligado a problemáticas emocionales más que a factores perceptivos y cognitivos que generan activaciones emocionales que inciden en la conducta y las expectativas de éxito tal como sostienen Brandão et al. (2011).

\section{Aplicaciones prácticas}

Este estudio permite determinar que un árbitro que dirige de manera exitosa un partido de fútbol cuenta con el desarrollo de ciertos factores psicológicos que influyen en su actuación, por lo cual el entrenamiento mental surge como una necesidad en la preparación deportiva. Al respecto, según Prat, Flores y Carbonero (2013) resulta relevante que los árbitros de fútbol perfeccionen sus características psicológicas y de comunicación a partir de una preparación específica que les permita desarrollar su trabajo de forma óptima cumpliendo, a la vez, un rol formador y educador en el deporte.

En tal sentido, se reconoce la importancia que tiene el diseño de intervenciones que respondan a las necesidades sentidas por el agente deportivo en cuestión para facilitar un desempeño eficaz (Guillén y Feltz, 2011). Así, a la luz de lo reportado, el psicólogo debe desarrollar una labor de asesoría directa frente a aquellos temas indicados como relevantes en el desempeño arbitral, como por ejemplo el autocontrol y el manejo de las exigencias y presiones, tanto internas como externas, a que está sometido el árbitro (Brandão et al., 2011).

Por ende, el psicólogo deportivo debe proveer herramientas concretas para gestionar el modo, momento y forma de experienciar las situaciones que desafían el correcto desempeño arbitral diseñando estrategias de intervención apropiadas a dicho diagnóstico y que oriente el accionar hacia el objetivo principal planteado por los árbitros: el manejo de juego. Esta línea de intervención implica el trabajo de elementos ligados a la gestión emocional, el autocontrol, las estrategias de afrontamiento y el control atencional junto a otros contenidos expuestos en la literatura tales como la evaluación de las demandas del juego, el manejo motivacional de las decisiones y la planificación mental con el fin de optimizar el desempeño arbitral (Mascarenhas et al., 2005; Samuel, 2015).

A partir de ello, siguiendo lo planteado por González (2006), resulta necesario el proceso de creación y/o ajuste de estrategias de intervención que no resulten de la simple extrapolación del trabajo con deportistas, sino que refieran a este ámbito particular de participación deportiva, lo cual, además, permitiría la ampliación y validación de un nuevo campo de intervención y también el reconocimiento como especialista de alto rendimiento de la figura del árbitro. 
En ese contexto, emergen desafíos relevantes para el proceso de formación de árbitros en el campo del desarrollo de habilidades mentales. Al respecto, Villalobos, Ugarte y Guillén (2002) reportan que los temas citados por los árbitros como más interesantes para su formación son los referidos a la psicología (30.12\%), por sobre los temas de relaciones humanas (14.45\%), medicina del deporte (12.04\%), preparación física (10.84\%) y alimentación (8.43\%). Por ende, dichos autores sostienen que las carencias en la formación de los árbitros son el reflejo de la falta de investigación en torno al tema lo cual otorga una relevancia práctica a estudios como el aquí expuesto.

De tal modo, resulta esencial para los/las profesionales en Psicología del Deporte preparar el terreno para trabajar en base a las necesidades específicas del entorno en que se aplicará su labor, generando un vínculo entre lo que se debe trabajar y cómo se deben abordar estos aspectos para una intervención productiva, en este caso, en el ámbito del arbitraje (García-Naveira, 2018; Gilbourne y Richardson, 2006; Mathers y Brodie, 2015; Peris-Delcampo y Cantón, 2018).

En tal sentido, Blumenstein y Orbach (2014) a partir de la detección de las variables mentales influyentes en el arbitraje desarrollaron y evaluaron, en una muestra de 10 árbitros ( $M=28.8$ años de edad y $M=8.7$ años de experiencia) una rutina para distintos momentos del desempeño arbitral logrando un aumento de autoconfianza y enfrentamiento al estrés en los participantes.

Para Patiño y Alonso (2015) la figura del árbitro es un tema que requiere de más estudios centrados en analizar su formación, desarrollo y su aportación en materia educativa, en etapas de iniciación y en diferentes deportes. En dicho contexto, si bien el tamaño de la muestra empleada en el presente estudio no permite generalizar los resultados deja abierta la puerta a futura investigaciones que aumenten el número de árbitros participantes, diferencien entre árbitros según experticia y experiencia (futbol internacional, nacional, formativo, amateur, femenino, etc.) y reporten aportes de los equipos multidisciplinarios que trabajen con los árbitros para contribuir a una mejor comprensión y perfeccionamiento de su labor.

\section{Referencias}

Abbedaim, A., Sebbane, M., Zitouni, A., Zubiaur, M. y Boumesdji, A. (2016). Efecto de la base de conocimientos y de la memoria experta en una tarea de detección de errores de arbitraje en balonmano. Revista Iberoamericana de Psicología del Ejercicio y el Deporte, 17, 239-245.

Al-Haliq, M., Lufti, Z. y Oudat, M. (2014). Levels of burnout among sports referees in Jordan. Journal of Physical Education and Sport, 14, 47-51.
Alonso-Arbiol, I., Arratibel, N. y Gómez, E. (2008). La motivación del colectivo arbitral en fútbol: un estudio cualitativo. Revista de Psicología del Deporte, 17(2), 187-203.

Anshel, M., Kang, M. y Jubenville, C. (2013). Sources of acute sport stress scale for sports officials: Rasch calibration. Psychology of Sport and Exercise 14, 362-370. https://doi.org/10.1016/i. psychsport.2012.12.003

Bohórquez, R. y Checa, I. (2017). Diseño y validez de contenido de una entrevista para la evaluación psicológica de porteros de fútbol. Revista de Psicología Aplicada al Deporte y al Ejercicio Físico, 2, E3. https://doi.org/10.5093/rpadef2017a3

Blumenstein, B. y Orbach, I. (2014). Development of psychological preparation program for football referees: Pilot Study. Sport Science Review, 23(3-4), 113-125. https://doi.org/10.2478/ssr2014-0007

Brandão, R., Serpa, S., Krebs, R., Araújo, D. y Machado, A. (2011). El significado de arbitrar: percepción de jueces de fútbol profesional. Revista de Psicología del Deporte, 20(2), 275-286.

Brewer, B., Vose, J., Van Raalte, J. y Petitpas, A. (2011). Metaqualitative reflections in sport and exercise psychology. Qualitative Research in Sport, Exercise and Health, 3, 329-334. https://doi. org/10.1080/2159676X.2011.607180

Di Corrado, D., Pellarin, E. y Agostini, T. (2011). The phenomenon of social influence on the football pitch: Social pressure from the crowd on referees' decisions. Review of Psychology, 18, 33-36.

Froeseler, M., Monteiro, A. y Mansur-Alves, M. (2018). Estresse e Coping de Árbitros de Futebol no Teste Físico FIFA. Psicologia: Ciência e Profissão, 38, 102-115. https://10.1590/1982-3703003492016

García-Naveira, A. (2018). Pasado, presente y futuro del psicólogo del deporte en el fútbol español. Revista de Psicología Aplicada al Deporte y al Ejercicio Físico, 3, E2. https:/doi.org/10.5093/rpadef2018a8

García-Santos, D., Vaquera, A., Calleja-González, J., González-Espinoza, S. e Ibañez, S. (2017). Estrés y técnica de arbitraje en baloncesto en función del género. Revista de Psicología del Deporte, 26(suplemento), 51-57.

Gilbourne, D. y Richardson, D. (2006). Tales from the field: Personal reflections on the provision of psychological support in professional soccer. Psychology of Sport and Exercise, 7, 325-337. https://doi.org/10.1016/j.psychsport.2005.04.004

González, J. (2006). Psicología aplicada al árbitro de fútbol: Características psicológicas y su entrenamiento. Sevilla, España: Wanceulen.

González, M., Selva, C. y Torregrossa, M. (2018). Mujeres y árbitras: historias de vida de una doble minoría en el deporte. Revista de Psicología del Deporte, 27(2), 23-30.

Guillén, F. y Feltz, D. L. (2011). A conceptual model of referee efficacy. Frontiers in Psychology, 2, 1-5. https://doi.org/10.3389/ fpsyg.2011.00025

Guillén, F. y Jiménez, H. (2001). Características deseables en el arbitraje y el juicio deportivo. Revista de Psicología del Deporte, 10(1), 23-34.

Guillén, R., Peñarrubia, C., Montero, J. y Adell, J. (2014). Metodología cualitativa aplicada a las ciencias del deporte. Zaragoza, España: Prensas de la Universidad de Zaragoza.

Heinemann, K. (2008). Metodología de la investigación empírica aplicada a las Ciencias del Deporte. Barcelona, España: Paidotribo 
Márquez, R. (2005). La dinámica de interacción del fútbol: la actuación de un árbitro y su contexto. Apunts de Educación Fisica y Deportes, 87, 83-89.

Mascarenhas, D., Collins, D. y Mortimer, P. (2005). Elite refereeing performance: developing a model for sport science support. The Sport Psychologist, 19, 364-379. https://doi. org/10.1123/tsp.19.4.364

Mathers, J. y Brodie, K. (2011). Elite refereeing in professional soccer: A case study of mental skills support. Journal of Sport Psychology in Action, 2, 171-182. https://doi.org/10.1080/2152070 4.2017.609018

Molina, P., Villamón, M. y Úbeda, J. (2015). La investigación cualitativa en las revistas españolas de ciencias del deporte (20072011). Revista de Psicología del Deporte, 24(1), 29-36.

Patiño, O. y Cañadas, M. (2015). Análisis de la figura del árbitro deportivo y su intervención en el proceso de formación deportiva. Revista Pedagógica Adal, 30, 25-32.

Peirooz, S. (2013). A consideration of the factors influencing soccer referees' judgment? an overview. International Journal of Academic Research in Business and Social Sciences, 3(5), 678685.

Pedrosa, I. y García-Cueto, E. (2015). Aspectos psicológicos en árbitros de élite: ¿afecta el salario a su bienestar emocional? Revista de Psicología del Deporte, 24(2), 241-248.

Peris-Del Campo, D. y Cantón, E. (2018). El perfil profesional del especialista en psicología del deporte en fútbol. Revista de Psicología Aplicada al Deporte y al Ejercicio Físico, 3, E9. https:/doi. org/10.5093/rpadef2018a6

Pla-Cortés, J., Gomá-i-Freixanet, M. y Avilés-Antón, O. (2015). Perfil de personalidad de los árbitros de baloncesto españoles en comparación con la población general. Revista de Psicología del Deporte, 15(2), 87-94. https://doi.org/10.4321/S157884232015000200010

Prat, M., Flores, G. y Carbonero, L. (2013). El rol del árbitro y su implicación en el fomento del juego limpio. Análisis y propuestas de intervención en el contexto de deporte universitario. Retos. Nuevas tendencias en Educación Física, Deportes y Recreación, 24(2), 72-78.

Ramírez, A., Alonso-Arbiol, I., Falcó, F. y López, M. (2006). Programa de intervención psicológica con árbitros de fútbol. Revista de Psicología del Deporte, 15(2), 311-325.

Rosado, A., Araujo, D., Mesquita, I., Correia, A., Mendes, F. y Guillén,
R. (2014). Perceptions of Fitness Professionals regarding Fitness Occupations and Careers: A Phenomenological Analysis. Revista de Psicología del Deporte, 23(1), 23-31.

Samuel, R. (2015). A Psychological Preparation Framework for Elite Soccer Referees: A Practitioner's Perspective, Journal of Sport Psychology in Action, 6(3), 170-187. https://doi.org/10.10 80/21520704.2015.1065938

Samuel, R., Galily, Y. y Tenenbaum, G. (2015). Who are you, ref? Defining the soccer referee's career using a change-based perspective. International Journal of Sport and Exercise Psychology, 15, 118-130. https://doi.org/10.1080/1612197X.2015.1079792

Schnyder, U. y Hossner, E. (2016). Psychological issues in football officiating: An interview study with top-level referees. Current Issues in Sport Science, 1(4), 1-14. https://dol.org/10.15203/ CISS_2016.004

Slack, L., Maynard, I., Butt, J. y Olusoga, P. (2013). Factors underpinning football officiating excellence: Perceptions of English premier league referees. Journal of Applied Sport Psychology, 25, 298-315. https://doi.org/10.1080/10413200.2012.726 $\underline{935}$

Tore, B. y Haugen, T. (2013). Anxiety level and decision-making among Norwegian top-class soccer referees. International Journal of Sport and Exercise Psychology, 11(2). 215-226. https://doi.org/10.1080/1612197X.2013.773665

Unkelbach, C. y Memmert, D. (2008). Game management, context effects, and calibration: The case of yellow cards in soccer. Journal of Sport \& Exercise Psychology, 30, 95-109. https://doi. org/10.1123/jsep.30.1.95

Vela, D. y Arbinaga, F. (2018). Aplicación del listado de situaciones estresantes para el arbitraje (LISEA) en árbitros de fútbol. Revista Iberoamericana de Psicología del Ejercicio y el Deporte, 13, 281-287.

Villalobos, D., Ugarte, O. y Guillén, F. (2002). Situación actual y expectativas del árbitro de fútbol profesional. Kinesis, 33, 13-20.

Voight, M. (2009). Sources of stress and coping strategies of US soccer officials. Stress and Health, 25, 91-101. https://doi. org/10.1002/smi.1237

Weinberg, R. y Richardson, P. (1990). Psychology of officiating. Champaign, IL, Estados Unidos: Leisure Press.

Wolfson, S. y Neave, N. (2007). Coping under pressure: Cognitive strategies for maintaining confidence among soccer referees. Journal of Sport Behavior, 30, 232-247. 


\section{Anexo 1}

\section{Pauta de Entrevista}

\section{Antecedentes demográficos/Datos generales}

- Nombre

- Edad

- Estado civil, composición familiar

- Tiempo de experiencia arbitral

- Tiempo como árbitro FIFA

- Descripción de su carrera como árbitro

2. Percepción general de la influencia del factor mental en el arbitraje

- ¿Qué importancia le da al factor psicológico en el arbitraje?

- ¿Cuán influyente es el factor psicológico en el arbitraje?

- (Si siente que influye) "¿De qué manera influye lo psicológico en la labor del árbitro
3. Identificación de los factores psicológicos considerados relevantes para el arbitraje

- ¿Qué factores psicológicos son los más relevantes para el arbitraje?

- ¿Por qué los considera relevantes?

4. Significados asociados a las variables mentales identificadas

- (Para cada variable mencionada) ¿Qué significa para usted la variable... ?

5. Impacto de los factores mentales identificados sobre el desempeño del árbitro

- ¿De qué forma concreta influye el factor... sobre el desempeño arbitral?

- ¿En qué situaciones se presentan estos factores?

- ¿Qué efectos tiene este impacto en tu desempeño?

- ¿Cómo te das cuenta de la influencia de este factor sobre tu trabajo? 